\title{
Pharyngeal Dystonia Misdiagnosed as Cricopharyngeal Dysphagia Successfully Treated by Pharmacotherapy
}

\author{
Ho Eun Park, MD, Myung Jun Shin, MD, PhD, Je-Sang Lee, MD, PhD*, Yong Beom Shin, MD, PhD \\ Department of Rehabilitation Medicine, Pusan National University School of Medicine-Biomedical Research Institute, \\ Pusan National University Hospital, Busan, Korea
}

\begin{abstract}
A 43-year-old woman suffered from drooling and dysphagia after a stroke in the left posterior inferior cerebellar artery territory. Videofluoroscopic swallowing study showed compatible findings of cricopharyngeal dysphagia. Despite the injection of botulinum neurotoxin, no symptom improvement was achieved and pharyngeal dystonia was considered as the cause. Medications for dystonia dramatically helped with saliva control and resulted in a small improvement in the progression of food from the pharyngeal to esophageal phase. After adjusting the drug dose, the patient was able to perform social activities without drooling. Moreover, she could consume food orally; however, this was limited to small amounts of liquid, and the main method of nutrition support was via an orogastric tube. Therefore, we suggest that physicians should make a differential diagnosis of combined dystonia in patients complaining of dysphagia by esophageal manometry and electromyography.
\end{abstract}

Keywords Deglutition disorders, Dystonia, Drug therapy

\section{INTRODUCTION}

Dysphagia after stroke is a common symptom reported in $28 \%-65 \%$ of patients [1]. As various parts of the brain are involved in swallowing, clinical manifestations of swallowing disorder vary [2].

Cricopharyngeal dysphagia (CPD) is a swallowing disorder caused by an inappropriate constriction of the cri- copharyngeal muscle, and the medulla is the most causative location [3].

To date, CPD treatment is focused on the relaxation of the cricopharyngeus muscle, for which methods such as injection of botulinum neurotoxin (BoNT), balloon dilatation, and myotomy are often used [4]. Meanwhile, little is known about pharmacotherapy.

Apart from CPD, inappropriate relaxation of the pha-

Received August 23, 2018; Accepted November 28, 2018

Corresponding author: Je-Sang Lee

Department of Rehabilitation Medicine, Gimhae Hansol Rehabilitation \& Convalescent Hospital, 2379 Gimhae-daero, Gimhae 50924, Korea. Tel: +8251-240-7485, Fax: +82-51-247-7485, E-mail: drleejs@gmail.com

*Current affiliation: Gimhae Hansol Rehabilitation \& Convalescent Hospital

ORCID: Ho Eun Park (http://orcid.org/0000-0002-4254-0596); Myung Jun Shin (http://orcid.org/0000-0003-4010-0383); Je-Sang Lee (http://orcid. org/0000-0001-6617-3113); Yong Beom Shin (http://orcid.org/0000-0001-5026-1696).

(c) This is an open-access article distributed under the terms of the Creative Commons Attribution Non-Commercial License (http://creativecommons.org/ licenses/by-nc/4.0) which permits unrestricted noncommercial use, distribution, and reproduction in any medium, provided the original work is properly cited. Copyright $\odot 2019$ by Korean Academy of Rehabilitation Medicine 
ryngeal muscle, called pharyngeal dystonia, also occurs and is often reported in cases of lesions in the cerebellum rather than the basal ganglia [5]. However, dystonia localized only in the pharyngeal muscle is extremely rare, and its pathogenesis is unclear and scarcely reported. Some studies have reported dysphonia and dysphagia due to pharyngeal dystonia, but a treatment strategy has not yet been established [6].

Herein, we report a rare case of improvement with pharmacotherapy in dysphagia caused by focal pharyngeal dystonia in a 43-year-old woman. We also discuss the mechanism of our successful pharmacological approach to treat dysphagia combined with dystonia.

Written informed consents were obtained.

\section{CASE REPORT}

A 43-year-old woman was admitted to the rehabilitation clinic due to severe drooling and dysphagia. One year and 2 months ago, she underwent coil embolization for subarachnoid hemorrhage due to left vertebral artery aneurysm rupture. Later, she was confirmed to have lesions of the pons, medulla, and cerebellum due to left posterior inferior cerebellar artery infarction detected by magnetic resonance imaging of the brain. However, a definite focal motor deficit was not observed on both the upper and lower extremities. The left upper and lower limbs showed slightly hyperactive deep tendon reflex compared to the right side. Pathological reflex was not elicited, and ataxia was not notable. Sensation and gag reflex were diminished, and hoarseness due to left vocal cord palsy was observed. However, issues in oromotor function were not suspected because pronunciation and articulation were clear without deviation of the tongue and uvula. Patient was taking aspirin and clopidogrel for the history of coil embolization and cerebellar infarction, and lansoprazole for the symptom of reflux.

Three months after onset, percutaneous endoscopic gastrostomy (PEG) was performed, and tubal feeding was continued. Despite aggressive rehabilitation therapy for dysphagia, such as neuromuscular electrical stimulation, there was no improvement.

Drooling, voice quality, and dysphagia resulted in significant quality of life (QOL) deterioration. Apart from the swallowing difficulty, she could independently perform activities of daily living, with 100 points on the Korean version of Modified Barthel Index but only 5 points on modified Teacher's Drooling Scale (mTDS). Her QOL was significantly compromised with moderate depression (19 points on Beck Depression Inventory [BDI]).

According to the videofluoroscopic swallowing study (VFSS) at 1 year and 2 months after onset, bolus progression was not achieved from the pharyngeal to the esophageal phase with severe food retention in the vallecula and pyriform sinus (67.5 points on the Functional Dysphagia Scale [FDS]). Since she was immediately referred to an experienced otolaryngologist and diagnosed with CPD with hypertonicity of upper esophageal sphincter (UES) after laryngoscopic examination, Botox (onabotulinumtoxin A; Allergan Inc., Irvine, CA, USA) injection (100 IU mixed in $5 \mathrm{~mL}$ of normal saline, 5 points, with $1 \mathrm{~mL}$ at each site) was administered under general anesthesia. However, symptom did not improve and still scored 67.5 points on FDS on VFSS that was conducted 2 weeks after the procedure.

Since the patient did not have underlying diseases or family history related to dystonia, the medical team presumed that the focal dystonia occurred after a stroke.

\section{Differential diagnosis}

On laryngoscopy, focal pharyngeal dystonia was confirmed rather than CPD, and esophageal manometry confirmed that the UES tone was significantly decreased.

\section{Treatment}

Pharmacotherapy was tested for the management of dystonia. Administration of basic treatment drugs for dystonia [7], Trihexin $2 \mathrm{mg}$ (trihexyphenidyl HCl; Tai Guk Pharm Co. Ltd., Seoul, Korea), Rivotril 0.5 mg (clonazepam; Roche Inc., Basel, Switzerland), and Neurontin 100 mg (gabapentin; Pfizer Inc., NY, USA) were started three times a day. One week after drug administration, mTDS showed significant improvement from 9 to 4 points. After 3 weeks, FDS remained at 61 points with no definite advancement; however, mTDS decreased to 2 points (Fig. 1A).

\section{Outcome and follow-up}

The patient wanted more controllable saliva secretion. Hence, doses of trihexyphenidyl were increased to $3 \mathrm{mg}$ three times a day. In addition, she complained of pain and discomfort with the PEG and hoped for oral feeding. In VFSS conducted 7 weeks after initiating medica- 
A

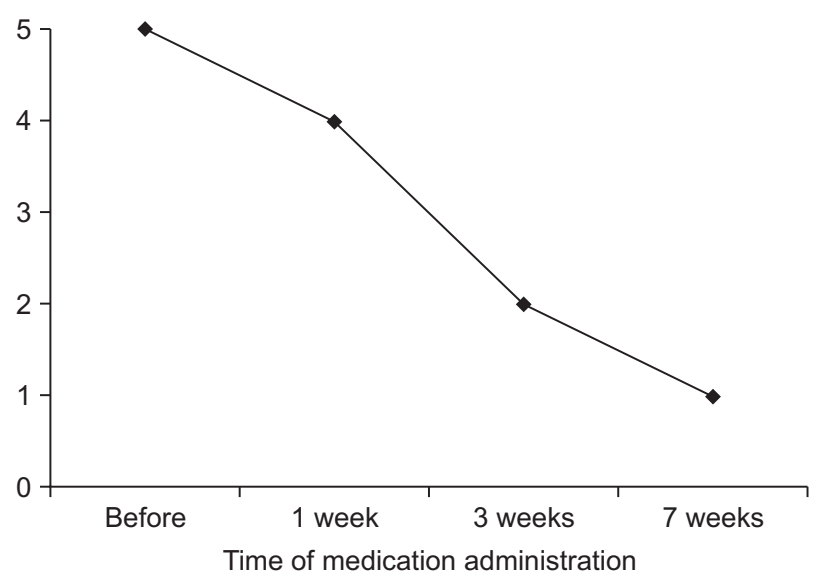

C

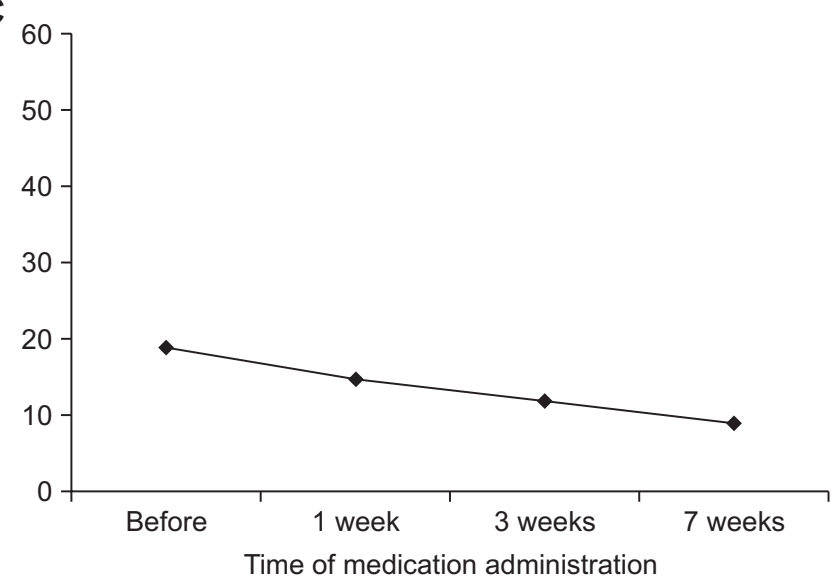

B

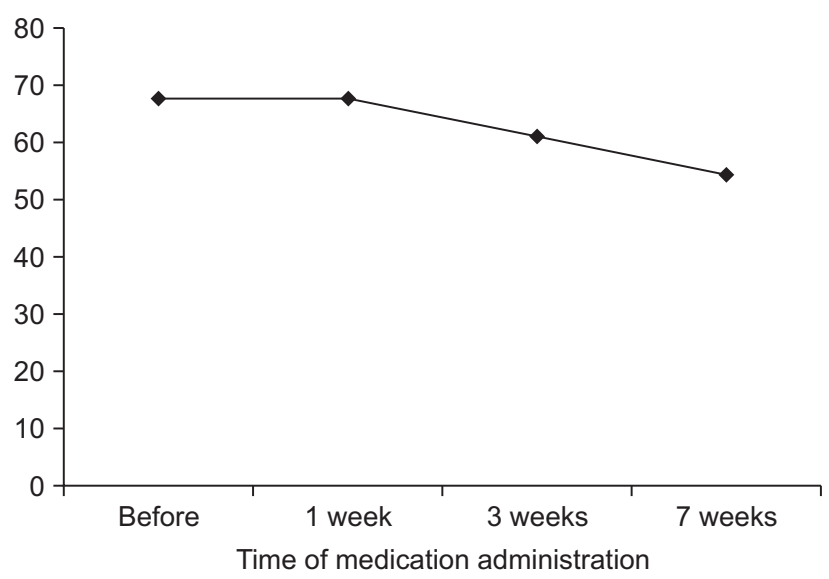

D

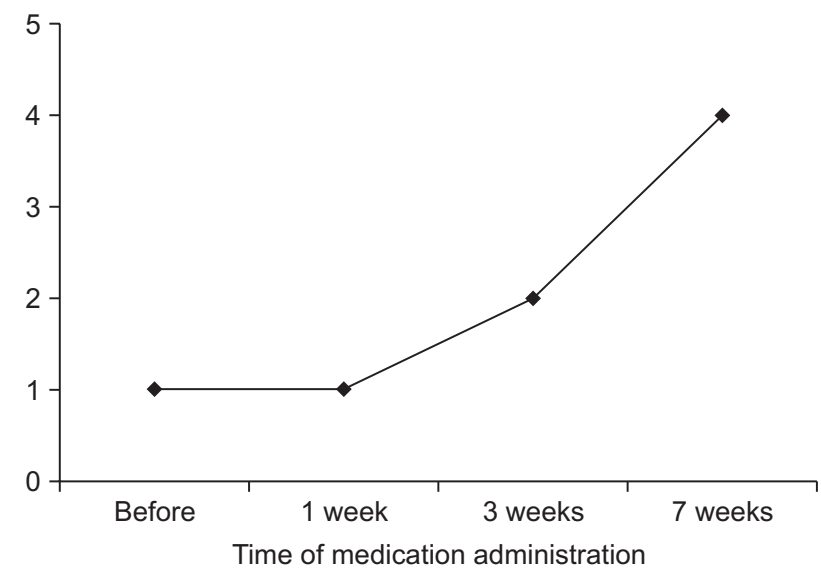

Fig. 1. Changes in indices according to administration of medication: (A) modified Teacher's Drooling Scale, (B) Functional Dysphagia Scale, (C) Beck Depression Inventory, and (D) Food Intake Level Scale.
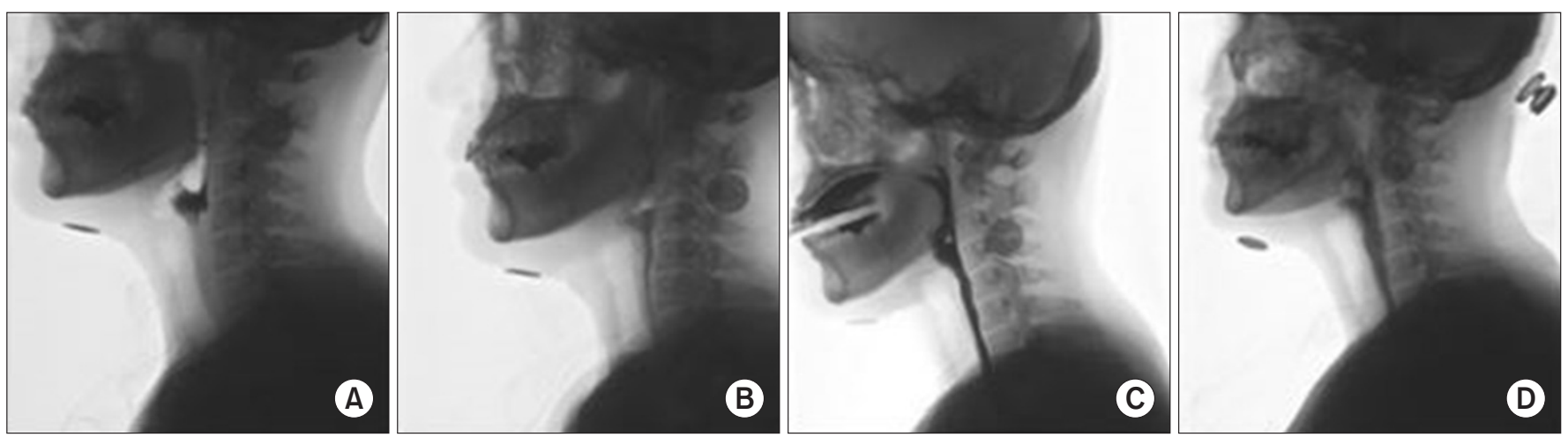

Fig. 2. Videofluoroscopic swallowing study according to the time of administration of medication: (A) before, (B) after 1 week, (C) after 3 weeks, and (D) after 7 weeks.

tion, oral administration of food was partially possible (Fig. 2). Although drooling was no longer observed and FDS was 54.5 points, oral feeding was extremely limited to minimal liquid which was insufficient to satisfy her nutritional requirement (Fig. 1B). Hence, tubal feeding using orogastric tube was tested. Since the gag reflex was weak and discomfort such as nausea during tube insertion was tolerable, compliance was high. The PEG tube was removed after she became proficient in using the orogastric tube, which significantly improved her QOL, and the BDI 
improved to 9 points (Fig. 1C).

Unfortunately, the patient failed to take her medication for 3 days due to the misplacement of medications. Symptom worsening after cessation of medication confirmed the effectiveness of drugs. Although further improvement of dysphagia has not been observed, the patient is currently on food intake level scale 4 , and treatment effects with pharmacotherapy were maintained for several months without worsening of symptoms or complications (Fig. 1D).

\section{DISCUSSION}

We present a rare case of a patient with dysphagia due to focal dystonia in the pharynx, which showed dramatic improvement with pharmacotherapy, despite the lack of symptom improvement for over 1 year from disease onset. The pathogenesis of focal pharyngeal dystonia after brain injury is unclear. VFSS is the gold standard for swallowing process evaluation, which visually confirms the flow of food. However, its limitation lies in cases where the bolus cannot progress through the UES, making it difficult to determine whether the cause is the UES itself or dysfunction of the muscle involved in the swallowing process. Misdiagnosis of CPD can be made with VFSS findings alone, leading to injection of BoNT to the cricopharyngeus muscle. In patients with medulla or pons lesions, swallowing disorders were particularly irrelevant with injection of BoNT [8], suggesting other causes of dysphagia in such patients. UES opening occurs by passive stretching due to active relaxation and laryngeal elevation of the cricopharyngeus muscle and expansion from food bolus formation [9]. Bolus flow is determined based on the contraction strength of the pharyngeal constrictor muscle and UES opening resistance. If any of the processes is unsuccessful, clinical symptoms of dysphagia such as CPD or pharyngeal dystonia may occur. Diagnosis of dystonia stems from suspicion of clinical features, making it difficult to confirm diagnostic or therapeutic approaches. Dystonia is often diagnosed and controlled simultaneously by oral medications and intramuscular injection of BoNT [10]. Although BoNT has been regarded as the first choice for focal dystonia, pharmacological treatment is considered as the next step if the former is ineffective.

In our case, CPD was suspected after VFSS findings and intramuscular BoNT injection to cricopharyngeus muscle was performed preferentially and successfully by an experienced otolaryngologist under generalized anesthesia. However, even after 2 weeks of BoNT injection, the patient's symptoms did not improve.

Esophageal manometry and electromyography were performed to evaluate the possibility of pharyngeal dystonia. In esophageal manometry, when maintaining lansoprazole for 1 week, hypopharyngeal intrabolus pressure was not detected by hypertonic, non-relaxed UES with decreased peristalsis of esophageal body. Electromyography also revealed hypertonicity of inferior pharyngeal constrictor muscle.

We considered the following possibilities to determine the cause of failure with BoNT injection. First, the most fundamental reason is the inappropriate selection of target muscle. The closure muscles of the upper esophageal sphincter include cervical esophagus, cricopharyngeus, and inferior pharyngeal constrictors. Among them, the striated cricopharyngeus muscle plays a major role. Inferior pharyngeal constrictor muscle causing pharyngeal dystonia was not targeted, because CPD was initially suspected and injection was performed only on cricopharyngeus muscle. Secondly, BoNT can only affect the cholinergic pathway, 1 of the mechanisms known to be involved in dystonia, and no other mechanism has been controlled which is discussed below.

After confirming the evidence of pharyngeal dystonia, additional BoNT injection to the inferior pharyngeal constrictor muscle was considered. Repeated injections were performed at intervals of several months because of the development of neutralizing antibodies against BoNT. In addition, the patient feared undergoing invasive treatments like injections or myotomy, and frequently complained of discomfort due to the gastrostomy tube. Therefore, oral medications were alternatively considered to manage pharyngeal dystonia.

Pharmacological approaches are subdivided into three major categories according to the mechanisms which affect the cholinergic system, $\gamma$-aminobutyric acid-ergic (GABAergic) system, and dopaminergic system. Administration of trihexyphenidyl, clonazepam, and gabapentin resulted in improvement of drooling and dysphagia without adverse drug reaction. Trihexyphenidyl, an anticholinergic drug, might act as an antagonist to postsynaptic M1 receptors, reducing the secretion of saliva. Clonaz- 
epam, a benzodiazepines, binds to $\mathrm{GABA}_{\mathrm{A}}$ receptors, facilitating inhibitory neurotransmission. However, the role of gabapentin, a GABA agonist, in pathophysiology of dystonia still remains unclear [7]. From the perspective of rehabilitation medicine, significant improvements in depression and dysphagia were noted after the patient received medication.

In conclusion, if CPD due to central lesion is suspected, focal pharyngeal dystonia should be considered. For the evaluation of swallowing difficulty caused by dystonia, laryngoscopic examination, esophageal manometry, and electromyography, in addition to VFSS, should be performed to clearly identify the tone of the cricopharyngeus and inferior pharyngeal constrictor muscles. We propose complementary analysis of results from these tests to improve the accuracy of the diagnosis. If tests suggest non-typical CPD such as focal pharyngeal dystonia, dysinnervation of the central nervous system and pharmacotherapy other than BoNT injection or surgery should be considered.

\section{CONFLICT OF INTEREST}

No potential conflict of interest relevant to this article was reported.

\section{ACKNOWLEDGMENTS}

This work was supported by the clinical research grant from Pusan National University Hospital in 2018.

\section{AUTHOR CONTRIBUTION}

Conceptualization: Shin YB. Writing - original draft: Park HE, Lee JS. Writing - review and editing: Park HE, Lee JS, Shin MJ, Shin YB. Approval of final manuscript: all authors.

\section{REFERENCES}

1. Cohen DL, Roffe C, Beavan J, Blackett B, Fairfield CA, Hamdy S, et al. Post-stroke dysphagia: a review and design considerations for future trials. Int J Stroke 2016;11:399-411.

2. Hamdy S, Aziz Q, Rothwell JC, Crone R, Hughes D, Tallis RC, et al. Explaining oropharyngeal dysphagia after unilateral hemispheric stroke. Lancet 1997;350:68692.

3. Steinhagen V, Grossmann A, Benecke R, Walter U. Swallowing disturbance pattern relates to brain lesion location in acute stroke patients. Stroke 2009;40:19036.

4. Kocdor P, Siegel ER, Tulunay-Ugur OE. Cricopharyngeal dysfunction: a systematic review comparing outcomes of dilatation, botulinum toxin injection, and myotomy. Laryngoscope 2016;126:135-41.

5. Ichihara N, Ichihara SI, Fujii S, Touge T, Nishioka M. An assessment of dysphagia using videofluorography in Parkinson's disease and progressive supranuclear palsy. Rinsho Shinkeigaku 2000;40:1076-82.

6. Shi LL, Simpson CB, Hapner ER, Jinnah HA, Johns MM 3rd. Pharyngeal dystonia mimicking spasmodic dysphonia. J Voice 2018;32:234-8.

7. Termsarasab P, Thammongkolchai T, Frucht SJ. Medical treatment of dystonia. J Clin Mov Disord 2016;3:19.

8. Lee WK, Seo HG, Seong MY, Yeom J, Lee WH, Han TR, et al. The effect of balloon dilatation and/or botulinum toxin injection on the severe dysphagic patients with cricopharyngeal dysfunction: case series. J Korean Dysphagia Soc 2017;7:69-75.

9. Sivarao DV, Goyal RK. Functional anatomy and physiology of the upper esophageal sphincter. Am J Med 2000;108 Suppl 4a:27S-37S.

10. Skogseid IM. Dystonia: new advances in classification, genetics, pathophysiology and treatment. Acta Neurol Scand Suppl 2014;(198):13-9. 